\title{
In-situ Low Energy Argon Ion Source for Artifact Free High Resolution STEM Imaging
}

\author{
$\underline{\text { Mikhail Dutka }^{1}, \text { Romaine Isaacs }^{2}, \text { Anna Prokhodtseva }^{1} \text {, and Tomáš Vystavěl }}{ }^{2}$ \\ 1. Thermo Fisher Scientific, Achtseweg Noord 5, 5651 GG Eindhoven, Netherlands \\ 2. Thermo Fisher Scientific, Vlastimila Pecha 12, 62700 Brno, Czech Republic
}

An in-situ low energy Ar source module [1], $\mu$ Polisher, enables localized low energy ion polishing of sample surfaces in the chamber of the scanning electron microscope (SEM) or a DualBeam system. This technique enables the removal of hydrocarbon contamination, Ga implantation and the amorphous layer in TEM lamellae prepared by focused ion beam Ga FIB[2] to enable artifactfree high resolution STEM imaging.

Localized cleaning is achieved by navigating to the target lamella on a grid using the SEM and inserting a retractable gas delivery needle. The inert Ar gas flow is ionized by the electron beam inside the nozzle with bore size $\sim 30 \mu \mathrm{m}$ placed about $100 \mu \mathrm{m}$ away from the sample surface. The ions are accelerated towards the sample surface, biased in the range of 20 to $500 \mathrm{~V}$, where the cleaning occurs. The sample is typically exposed to the ion beam for 1-3 minutes, depending on the material composition and desired thickness removal. The cleaned region has a diameter $\sim 10$ to $20 \mu \mathrm{m}$, which is comparable to the size of most lamellae. Accurate targeting with the needle means that redeposition from the nearby grid can be avoided.

We have performed in-situ low energy Ar cleaning on several materials including: Sapphire (0001), $\mathrm{GaN}$, and STO, comparing the same region of interest before and after polishing. Figure 1 shows a GaN lamella cleaned with $\mathrm{Ar}^{+}$ions generated by a $1 \mathrm{kV}, 22 \mathrm{nA}$ electron beam and targeted at the sample by applying a stage bias of $-200 \mathrm{~V}$ for $60 \mathrm{sec}$. The improvement in surface quality is apparent from the removal of hydrocarbon contamination and the marked increase in the image contrast (images acquired with the same brightness and contrast parameters). shows high resolution TEM images of a STO lamella. The $3 \mathrm{~nm}$ amorphous damage layer at the edge of the lamella is removed after 1 minute cleaning on each side.

\section{References:}

[1] Mulders, J. and Trompenaars, P. 2016. An in-situ Low Energy Argon Ion Source for Local Surface Modification. European Microscopy Congress 2016: Proceedings. 453-454

[2] Prokhodtseva, A., Mulders, J., \& Vystavel, T. (2017). Applications of an in-situ Low Energy Argon Ion Source for Improvement of TEM and SEM Sample Quality. Microscopy and Microanalysis, 23(S1), 298-299. doi:10.1017/S1431927617002173 

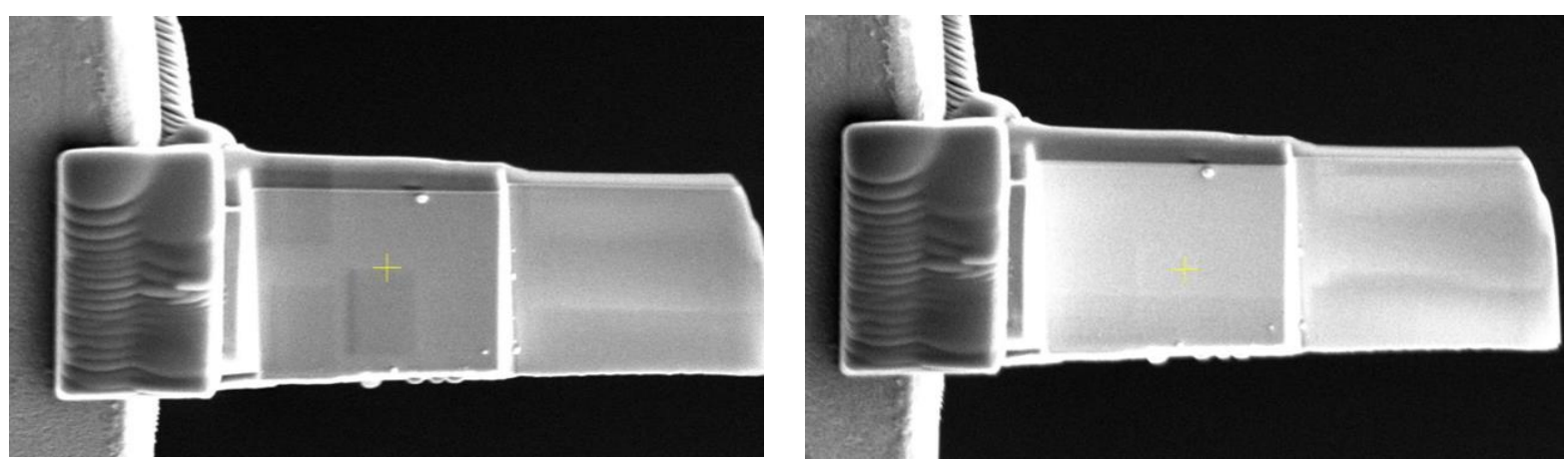

Figure 1. GaN Lamella before (left) and after (right) cleaning with low energy ion source with parameters 1kV, 22nA -200V stage bias. Imaged on a ThermoFisher Scientific Helios G4 CX, a marked contrast change on the lamella surface and contamination removal are immediately apparent.
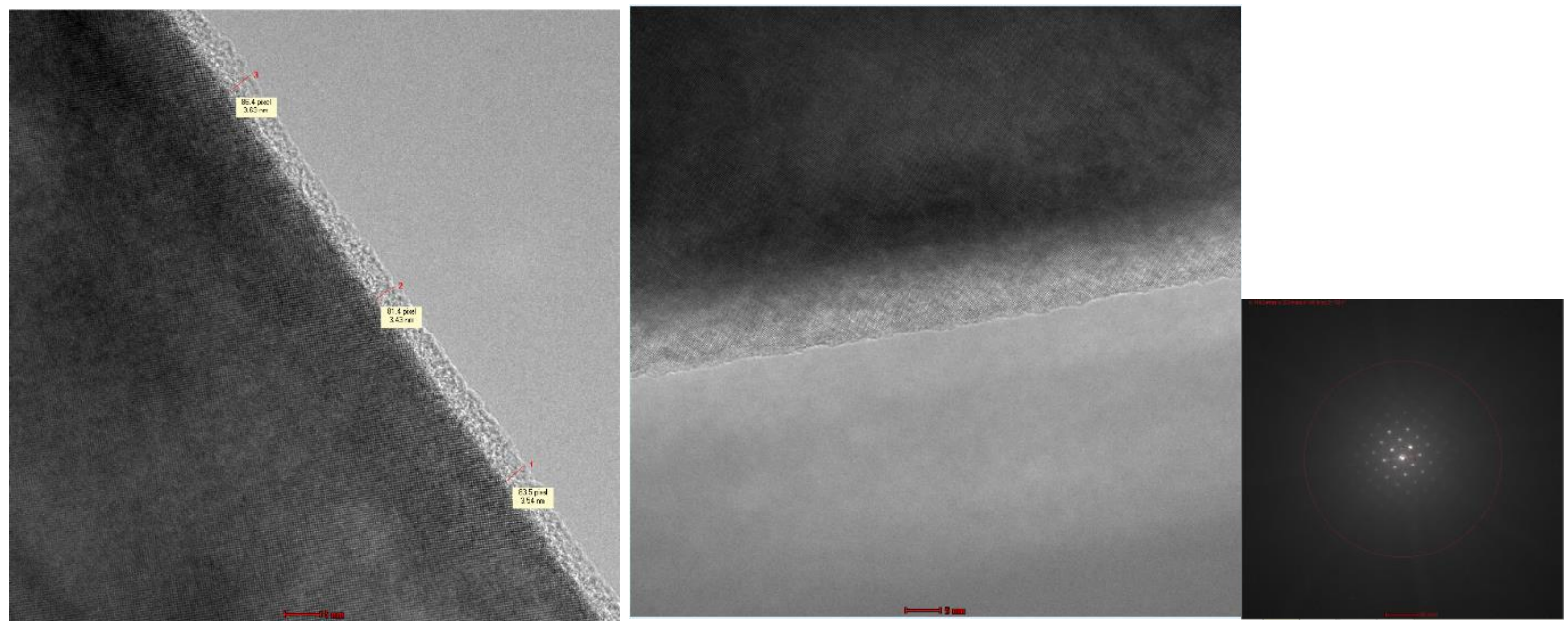

Figure 2. STO Lamella images at Magnification 650kx on ThermoFisher Scientific Talos F200 shows removal of the $3 \mathrm{~nm}$ amorphous layer damage the edge of the lamella 